Methods The operational research was a retrospective analysis of reported data from October to December 2016, 2017 and 2018. The data used was sourced from DHIS2.Upon assessment of completeness tabulation was done, and summary statistics obtained.

Results A total of 289,875 women who visited 1stANC in October to December 2016, 277,196 (95.6\%) were tested for HIV and12,161 (4.3\%) tested HIV positive, the proportion that was screened for syphilis was 211,546 (72.9\%) women and out of those,2,396 (1.1\%) women tested positive for syphilis. In October to December 2017, a total of 336,512 women visited ANC, 306,573 (91.1\%) women tested for HIV and 15,056 (4.9\%) turned positive while 262,567 (78\%) were tested for syphilis and 3,072 (1.2\%) turned positive. In 2018 same quarter, 336,687 women visited ANC, 298,598 (88.9\%) were tested for HIV, 16, $5805(6 \%)$ turned positive while 307,842 (91\%) tested for syphilis and 3,464 (1.1\%) tested positive of syphilis. Thus, for the first time ever in Kenya syphilis testing has caught up with HIV testing at $1^{\text {st }}$ ANC. This is an improvement to $91 \%$ from $73 \%$ which is critical for achievements of the country's eMTCT targets.

Conclusion Syphilis not only result $s$ in intrauterine fetal demise, but also facilitates HIV acquisition and impede progress towards eMTCT. Kenya's commitment to reduce MTCT of HIV and Syphilis to below 5\% by 2019 requires our country to improve Syphilis screening/testing. To increase syphilis testing, the use of dual testing kits at ANC should be used widely and every woman at ANC should be encouraged and educated on the importance of syphilis test.

Disclosure No significant relationships.

\section{P290 TEMPORAL DISCOUNTING AND ENGAGEMENT IN CARE AMONG HIV-INFECTED PREGNANT AND BREASTFEEDING WOMEN}

${ }^{1}$ Jessica Londeree Saleska*, ${ }^{2}$ Abigail Norris Turner, ${ }^{3}$ Maria Gallo, ${ }^{1}$ Marcel Yotebieng ${ }^{4}$ Abigail Shoben. ' Ohio State University College of Public Health, Epidemiology, Columbus, USA; ${ }^{2}$ Ohio State University, Internal Medicine, Infectious Diseases, Columbus, USA; ${ }^{3}$ The Ohio State University, Division of Epidemiology, Columbus, USA; ${ }^{4}$ Ohio State University College of Public Health, Biostatistics, Columbus, USA

\subsection{6/sextrans-2019-sti.411}

Background For pregnant or breastfeeding HIV-positive women, poor adherence to antiretroviral (ARV) medication and disengagement from care can increase risk of mother-tochild transmission of HIV. HIV-positive women exhibiting high temporal discounting (TD; defined as the tendency to discount future rewards relative to rewards received closer to the present) may be less likely to adhere to recommendations for the prevention of mother to child transmission (PMTCT).

Methods We performed a secondary analysis of data from a randomized controlled trial conducted in the Democratic Republic of Congo, which assessed the effects of a conditional cash transfer intervention among 433 newly-diagnosed HIVinfected pregnant women. We measured TD at baseline using a delay discounting task. We dichotomized TD (high/low) and examined associations between TD and uptake of available PMTCT services, retention in care and viral suppression at 6 weeks postpartum. We used log-binomial regression models to calculate unadjusted and adjusted risk ratios (RRs) for the effects of high vs. low TD on these outcomes.
Results At baseline, participants had a median age of 29 years, median of 10 years of education, and median gestational age of 26 weeks. The majority (86.6\%) of women exhibited high TD, where $13.4 \%$ exhibited low TD. High TD was associated with incomplete uptake of PMTCT services (adjusted RR: 1.64, 95\% confidence interval (CI): 1.08-2.50) and lower viral suppression at 6 weeks postpartum (adjusted RR: 0.77, 95\% CI: 0.66-0.90). Temporal discounting was not associated with retention to care in unadjusted (RR: 1.57 95\% CI: $0.78-3.15$ ) or adjusted analyses (RR: 1.56 , 95\% CI: $0.76-$ 3.18).

Conclusion Understanding the mechanisms underlying women' behavioral choices is crucial to optimize interventions for behavior change. Our results indicate that women exhibiting high temporal discounting represent a critical population for interventions to improve adherence and engagement in PMTCT care.

Disclosure No significant relationships.

\section{P291 IMPACT OF PMTCT SERVICE UPTAKE ON OUTCOME OF CARE AMONG WOMEN ATTENDING ANTI NATAL CARE IN NIGERIA}

${ }^{1}$ Chidiebere Ezeokafor*, ${ }^{2}$ Adaoha Anosike. ${ }^{1}$ National Agency for the Control of AIDS, Resource Mobilization and Performance Management, ABUJA, Nigeria; ${ }^{2}$ National Agency for the Control of AIDS, Nigeria, Research Monitoring and Evaluation, ABUJA, Nigeria

\subsection{6/sextrans-2019-sti.412}

Background Nigeria has the second largest global burden of HIV/AIDS and also contributes the largest proportion of new vertically acquired HIV infections among children. Despite the effort to control the HIV/AIDS epidemic, elimination of mother to child transmission remains a huge challenge. The study aimed to assess the effect of outcome of care on utilization of prevention of mother to child transmission (PMTCT) services.

Methods A multi stage cross sectional study was conducted in 11 states in Nigeria. A systematic random sampling was used to select 365 clients (women living with HIV) receiving PMTCT services in primary and secondary health facilities. Information on PMTCT and outcome of care were extracted using structured questionnaire while descriptive and bivariate data were analyzed using SPSS version 21.

Results Majority (84.6\%) of the clients received HIV counselling and testing at ANC clinics while 40.2\% were counselled in a group and $26.7 \%$ counselled individually. A high proportion $(93.8 \%)$ of clients were aware of the early infant diagnostic care services being offered while $92.2 \%$ were aware of Navirapin drugs being provided daily for 6 weeks at the health facilities. Similarly, majority $(90.0 \%)$ of the clients were aware that family planning services are provided in the health facilities while $93.8 \%$ had access to infant feeding counselling at ANC. There was no significant difference between outcome of care and utilization of PMTCT services. However, majority of clients who utilized PMTCT services had improved outcome of care.

Conclusion Increased uptake of PMTCT services at ANC contributed to improved outcome of care of pregnant living with HIV and their children. Effort should be made to ensure more women attend ANC to achieve elimination of mother to child transmission in Nigeria.

Disclosure No significant relationships. 